\title{
El paradigma abya-yalista, cuna del pensamiento indígena y la educación intercultural, otra visión de la orientación humana
}

\author{
Mónica Valencia Bolaños*
}

Recepción: 10 de marzo de 2016

Aceptación: $\mathbf{8}$ de noviembre de 2016

*Universidad Nacional de Educación, Ecuador Correo electrónico: mvalencia10@gmail.com

Se agradecen los comentarios de los árbitros de la revista.

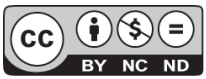

\begin{abstract}
Resumen. El abordaje conceptual de una educación intercultural desde el pensamiento indígena ecuatoriano a través del paradigma abya-yalista, es el objetivo de este trabajo. Asimismo, la aproximación a la cosmovisión del diálogo horizontal sumado al respeto, la corresponsabilidad social, las orientaciones del sabio anciano y el cultivo del hombre en respeto a la madre tierra son, entre otras aristas, las ideas básicas para profundizar en este pensamiento y develar la trascendencia de la escuela como el centro de su organización social. La metodología empleada fue la documental. Los resultados apuntan a lo axiológico y están dirigidos a la valoración de un modelo educativo originario que enfatiza en una orientación del buen vivir desde el ser para el convivir.
\end{abstract}

Palabras clave: Abya yala, educación intercultural, pueblos originarios, comunidad, orientación.

\begin{abstract}
Paradigm abya-yalista, Cradle of Indian Thought and Intercultural Education, Another View of Human Orientation

Abstract. The conceptual approach of an Intercultural Education, from the Ecuadorian indigenous thought through the abya-yalista paradigm (world in creation), is the objective of this research work. The approach to the world view of the horizontal dialogue added to respect, social co-responsibility, orientations of the wise elder, cultivation of the man in deference to the mother earth, are among other aspects, the basic ideas to deepen this thought and unveil the transcendence of the school as well as the center of their social organization. The methodology was a documentary investigation, and the results obtained are mainly the axiological ones directed to the valuation of an original educational model that emphasizes in an orientation of the good living from the being for the coexistence.
\end{abstract}

Keywords: Abya yala, intercultural education, indigenous people, community, orientation.

\section{Introducción}

Aquella visión de ver a los pueblos indígenas como pueblos bárbaros, idólatras, salvajes, primitivos, carentes de conocimiento y sin historia, fue justificante de los colonizadores para desatar una guerra de exterminio. Desde entonces, son más de quinientos años de resistencia. No obstante, estos seres supuestamente vacíos, ignorantes y atrasados se han mantenido con el tiempo, además de enfrentar como nadie una sistemática e implacable dominación, así el caso de los hermanos indígenas de la República del Ecuador.
Los pueblos y comunidades indígenas del territorio ecuatoriano no sólo se han mantenido en resistencia, sino que también se han salvaguardado en un trabajo silencioso, discreto y sistematizado. El proceso de visualización social logrado en la actualidad ha conservado a toda costa su identidad, sabiduría y racionalidad.

Ser indígena en territorio ecuatoriano representa al ser humano despojado, excluido, olvidado, de los beneficios y desarrollos de una política de Estado que los mantenía como último punto de su agenda. Sin embargo, desde una histórica riqueza connatural a la organización comunitaria, cuya raíz data de una 
ancestralidad cultural de más de veinte mil años, les permitió estructurar y rediseñar una racionalidad de vida nutrida de saberes, haceres, sentires y convivires que han hecho posible su vigencia y trascendencia como pueblos únicos orientados por sabios.

De allí que el propósito de esta investigación documental esté basado en una nueva mirada que da paso a la comprensión de la racionalidad en el pensamiento indígena, en una que conciba la educación intercultural como un paradigma de reflexiones civilizatorias que abren el diálogo de saberes. A pesar de constituir una temática por demás delicada, ya que las diversas aristas pluriculturales y multiétnicas constituyen la esencia de nuestra realidad social, entonces lo indígena se vuelve la respuesta necesaria para que el hecho social negado sea develado. De esta manera, la intencionalidad investigativa busca aproximarse a esas ideas y pensamientos de esos supuestos hombres "vencidos", pero con una historia que los hace dueños de una reserva moral y también parte de una escuela que por sí misma se suscribe a un paradigma llamado abya-yalista.

Dentro de esas ideas y pensamientos está la concepción que ha caracterizado a estos pueblos originarios del Ecuador, como lo es, la Escuela, que desde siempre ha constituido la respuesta comunitaria para la vida. Por medio de esta unidad organizacional buscan resolver algunos problemas concretos al interior de las comunidades: agua, salud, explotación de los comerciantes, servicios sociales, entre otros. Desde la escuela se dan las orientaciones para diseñar y ejecutar las formas de trabajo comunitarias tradicionales como la minga, el "presta manos", el consejo de los sabios; es decir, que la escuela constituye el centro de la organización y operacionalización indígena para el bienestar del colectivo.

La orientación que toma este legado indígena en el transcurso de los últimos siglos es para los fines requeridos por demás significativo, puesto que la trascendencia de sus aportes, constructos y logros son referentes orientados a la construcción de actitudes fundadas en un ethos que traduce pluriversidad intercultural: el respeto, admiración y reconocimiento a la capacidad del hombre en tomar la responsabilidad de su propio destino quien dentro de las más grandes adversidades ha logrado una educación que constituye un verdadero ejemplo de progreso, civilidad y autogestión.

A partir de ello, esta racionalidad propone la creación de un mundo nuevo, un mundo posible basado en el desarrollo de un nosotros comunitario en donde todos, indígenas y no indígenas, tengan cabida en el mundo del otro, con la concepción intercultural que se evidencia en una convivencialidad con un nuevo y amplio horizonte del sentido del buen vivir/vivir bien, proclamado por los pueblos indígenas de nuestra única América Latina.

\section{La insurgencia histórica y la configuración multiétnica}

Se considera como punto de partida la exploración de la historia para comprender el presente. Por esto es pertinente considerar el momento de la llegada de los conquistadores. A partir de este evento, dio inicio un proceso de dominación que ha dirigido a los pueblos originarios a una condición de marginalidad todavía presente en algunos de los países latinoamericanos, cuya población indígena está presente en la categoría poblacional de los más excluidos y olvidados por parte de los gobiernos en turno.

No se intenta analizar las causas, procesos y facetas de dicha invasión; sin embargo, sí se considera como fenómeno significante el exterminio no solamente de los recursos naturales y minerales, que fue en primera instancia el objetivo del conquistador, sino en esencia la eliminación de los contenidos simbólicos, de la sabiduría y prácticas para poder imponerse en las páginas de la historia de nuestro continente.

Según Guerrero Arias (1993), los pueblos indígenas, a pesar de las condiciones de opresión, violación y represión, no fueron las víctimas pasivas como algunas fuentes históricas los presentan. Sus raíces, cuyo rasgo esencial es comunitario, organizado, de liderazgo cruzado (vertical-horizontal), produjeron en ellos un espíritu de insurgencia que hizo posible la sobrevivencia a tal holocausto americano.

Es de notar que al citar insurgencia, no se refiere a una categoría teórica. Por el contrario, es indudable que la postura investigativa va más allá, es la constatación fehaciente del proceso de lucha que llevan y han llevado estos pueblos, pues desde su cotidianidad fueron fraguando respuestas contra el poder dominante.

Dentro de las respuestas gestadas, se encuentra precisamente el poder de enfrentar la hegemonía del poder del invasor al transformar esas estructuras impuestas por las que construirían la sociedad futura y demostrar que no sólo resistieron, sino que rebasaron la experiencia. El hombre y mujer del Reino de los Quitus (actual capital de los ecuatorianos), dueños y amos de sus territorios, estaban siendo vejados y a la vez reinventados como nuevos seres.

Entre esas innovaciones se inserta la arista de la multiculturalidad, ya que lo indígena no se agota a sí mismo. Es de considerar la configuración espacial y geográfica que hizo posible la diversidad en el ámbito social y étnico. Por esta razón, a partir de lo indígena, se puede entender también lo mestizo, lo criollo, lo cholo, lo negro y toda la gama de complejidades simbólicas que se reflejan en las distintas relaciones interétnicas. 
Partiendo de esta riqueza de tipologías, cabe entender la diversidad de nuestra configuración multiétnica, en cuyo reconocimiento se puede constatar la existencia de pueblos distintos, portadores de rasgos culturales diversos y cargados de identidades propias, únicas, con una belleza integral, y con otra orientación hacia el valor del hombre, desde sus talentos y potencialidades.

Flores Galindo (1988) afirma que sólo a partir de la diversidad latinoamericana se puede superar la connotación discriminatoria, término que nace de un error de apreciación del imaginario de Colón, hacer que recupere la historicidad de un proceso milenario, y repensar en lo indígena en términos de civilización, tan igual a otras culturas como los griegos o chinos, a fin de sobrepasar las abstracciones, desprenderse de las mitificaciones y aproximarse al saber ancestral que constituye uno de los pilares de nuestra cultura actual.

\section{Diversidad paradigmática}

Para explorar la diversidad de pensamientos, ideas y posturas, se toma las síntesis de García (2011), quien plantea la relación entre interculturalidad y paradigmas, y, más aún, cómo cada una de estas perspectivas implica no sólo una concepción de cultura, sino sobre todo una orientación hacia reflexionar y hacer interculturalidad. García (2011) menciona a Morín desde su planteamiento referido a la existencia de dos grandes paradigmas: de occidente y de oriente. Al interior del paradigma de occidente se pueden desplegar dos grandes perspectivas paradigmáticas en disputa: fragmentación y complejidad. Respecto a ésta última, señala:

Proponemos la siguiente definición: un paradigma contiene, para cualquier discurso que se efectúe bajo su imperio, los conceptos fundamentales o las categorías rectoras de inteligibilidad al mismo tiempo que el tipo de relaciones lógicas de atracción/repulsión (conjunción, disyunción, implicación u otras), entre estos conceptos o categorías. De este modo, los individuos conocen, piensan y actúan en conformidad con paradigmas culturalmente inscritos en ellos. Los sistemas de ideas están radicalmente organizados en virtud de los paradigmas (García, 2011: 218).

Esto es, que desde las percepciones, consensos y acuerdos conscientes o inconscientes, los hombres comparten criterios y consideraciones que rigen sus modos de ser, sentir y actuar como propios modos representativos de la cultura a la que pertenecen, como al paradigma vigente consensuado.
Por otra parte, desde la perspectiva de fragmentación, la cultura será mirada, reflexionada y actuada como un fragmento, troceada respecto de un todo mayor. Es vista como la pieza de una gran máquina en donde se pueden cambiar las piezas, pero nunca se pondrá en cuestionamiento la máquina misma. Separa al interior de la cultura el sujeto del objeto, la razón de la intuición y el pensar del hacer. Situados en esta perspectiva, muchas veces ni siquiera se le da a las otras culturas valor de tales criterios.

Desde la perspectiva de la complejidad, la cultura será vista en sus múltiples interrelaciones, en donde se procure descifrar lo que emerge de ese conjunto interrelacionado más que sus partes. Se buscará una visión sistémica de la cultura, desde su dinámica relacional, como un todo complejo organizado en relación con otras culturas consideradas como tales.

En el primer caso, la interculturalidad será mirada y actuada, a lo sumo como culturas manifiestas, momentos, elementos, piezas, fragmentos poco interrelacionados entre sí. En el segundo caso, como culturas dinámicas profundamente interrelacionadas unas con otras, co-constructoras de realidad, co-generadoras de modos de vida más o menos con-convivenciales.

Desde el paradigma oriental, prosigue García (2011), las culturas son consideradas como una maya (término hindú alusivo a una imagen irreal), es decir como una ilusión. Las culturas miradas y reflexionadas como obra de teatro, como un acto de magia, como obra de un mago invitan a reflexionar y actuar una irrealidad figurada. El mundo, sus culturas, todo, es una gran ilusión en la que todos participamos en el juego de hacernos creer unos a otros que esta ilusión es real.

Las culturas son un engaño, un juego de máscaras. Lo que pensamos, vemos, hacemos, es parte de este engaño colectivo, pues en realidad todo lo que nos rodea es una maya. Vivimos permanentemente ilusionados, engañados, enmascarados, creyendo que nuestra cultura es la verdadera, la plena, la cual es una afirmación de todas las culturas, y entonces se encuentra en el juego de las culturas enmascaradas, en el drama o el teatro de la vida, la propia tierra y el cosmos son máscaras del gran teatro cósmico.

Sin embargo, García (2011), autoridad directiva de la Universidad Indígena Amawtay Wasi en el Ecuador, propone otro paradigma, el Abya-yala (en lengua kuna: tierra en plena madurez). La idea de cultura se vuelve más compleja aún, pues las interrelaciones, la vincularidad y la reciprocidad se da entre todos los elementos de la vida, en donde las culturas se implican unas a otras y a todos los otros conceptos que están presentes en cada una de ellas: el aire, la tierra, el agua, el clima, el paisaje, el cosmos; cada uno de ellos es pensado y actuado como aspecto sustantivo de la interculturalidad. 
Desde este paradigma lo que se trata es develar esa orientación originaria que consiste en criar la interculturalidad al igual que los seres humanos. La interculturalidad tiene que ser co-generada, co-construida, criada de manera complementaria, recíproca, proporcional y correspondiente en vínculo con el conjunto de elementos que entran en juego en la crianza. La cultura también nos cría: es parte de la sabiduría con la cual el cosmos vivo y nosotros como parte de él nos vamos autocriando.

Asimismo, se hace referencia a la perspectiva paradigmática de la negritud. Lo que se está proponiendo es la construcción de nuevos marcos epistemológicos que incorporan y negocian conocimientos occidentales y conocimientos no occidentales, indígenas, pero también negros (y sus bases teóricas y vivenciales, pasados y presentes). Busca mantener siempre como fundamental la necesidad de enfrentar la colonialidad del poder a la que estos conocimientos han sido sometidos. Marcos epistemológicos que pluralizan, problematizan y desafían la noción de un pensamiento y conocimiento totalitario, único y universal desde una postura política y ética, abren la posibilidad y el reconocimiento de distintos modos de pensar.

Es de resaltar que esta otra perspectiva del paradigma abyayalista conlleva a una postura de una "interculturalidad global, cuya lógica representa otra forma de pensar y una práctica que trabaja en los límites de los conocimientos indígenas y negros, traduciendo los conocimientos occidentales a las perspectivas indígenas y negras del saber, y a sus necesidades políticas y concepciones éticas" (Walsh, 2003: 17).

Sin duda Walsh (2003), directora del doctorado en Estudios Culturales Latinoamericanos de la Universidad Andina Simón Bolívar, sede Ecuador, un cambio de paradigma es un proceso perturbador que modifica los núcleos organizadores de la sociedad, la civilización, la cultura y la noósfera (como pensamiento colectivo). En otras palabras, un nuevo paradigma implica una orientación transformadora del modo de pensamiento, del mundo del pensamiento y del mundo pensado. Cambiar el paradigma es a la vez cambiar de creencia, de ser y de universo.

Con estas reflexiones se fortalecen los hilos conductores a la crítica del proyecto medular de la modernidad (paradigma occidental), el cual postulaba al conocimiento científico como la única fórmula válida de producir verdades sobre la vida humana y la naturaleza. Es de notar que como un conocimiento que se crea universalmente oculta, invisibiliza y silencia las otras epistemes, como también lo hacen con quienes producen otro conocimiento. El episteme indígena ha sobrevivido a esta realidad.

\section{Concepción de lo intercultural}

En los últimos años ha surgido una adopción un tanto desmedida de algunos gobiernos latinoamericanos con fragmentos del discurso indigenista respecto a la interculturalidad, que a la vez se inserta, en la construcción ideológica de políticas del buen vivir/vivir bien, o en todo lo que se refiere a la educación en diversidad.

Dentro de este contexto, surge la necesidad de discutir nociones de interculturalidad, pedagogía intercultural, políticas públicas interculturales, sociedad intercultural, entre otros, por lo que delimitar este constructo es fundamental para el hilo discursivo de esta investigación. En este sentido, se considera la posición de Moya (2009: 22), destacada académica ecuatoriana, y quien afirma que:

como ocurre en otras nociones, la de interculturalidad no se ha desarrollado ni asimilado de manera homogénea, y es precisamente en los núcleos de poder indígena continental (refiere a Mesoamérica y los Andes), donde éstas son más controversiales y simultáneamente donde se proponen más soluciones y sentidos.

A partir de lo anterior, se deduce que esa diversidad de significados marca una denotada ambigüedad a la conceptualización y funcionalidad de la interculturalidad y lo intercultural. Sin embargo, Moya (2009) asevera que hay ciertos elementos comunes cuando se refieren a ámbitos como educación, salud y servicios institucionales vinculados a un nuevo posicionamiento, reconocimiento y visualización de lo indígena frente a un diálogo con lo no indígena. Es más, la esencia de la interculturalidad desde lo indígena marca pauta clarísima del colectivo, es decir, con una visión integracionista promovida por siempre desde el paradigma abya-yalista, y poco comprendida por los estados occidentalizados, mientras que García (2011: 14) admite la diversidad de culturas "subrayando su diferencia y proponiendo políticas relativistas de respeto que a menudo refuerzan la segregación”. La interculturalidad es "la confrontación y entrelazamiento que sucede cuando los grupos entran en intercambios".

De allí que su distinción entre ambos términos y conceptos sería en cuanto a que la multiculturalidad supone la aceptación de lo heterogéneo y la interculturalidad considera que "los diferentes" son lo que son en relaciones de negociación, conflictos y préstamos recíprocos; involucra las diversas acepciones en torno a la interculturalidad, es decir, las diferencias y las fusiones, la inclusión y la exclusión, la conexión o desconexión como parte de su dinámica en la globalización marcada por el capitalismo y neoliberalismo de nuestros tiempos. 
Asimismo, Sarango (2010) se aproxima de mejor manera a la concepción de una interculturalidad desde la vivencia de los pueblos indígenas en los últimos quinientos años. Él no sólo aporta una nueva concepción al respecto, sino que propone una tipología con base en esas vivencias históricas, incluida la que ha experimentado en carne propia como dirigente indígena ecuatoriano. A continuación su propuesta:

a) Interculturalidad subordinada: es la que han vivido los pueblos indígenas estos últimos quinientos años. Es la relación desigual entre culturas, los indígenas y no indígenas.

b) Interculturalidad institucionalizada: establecida en las leyes como respeto a las diferencias; sin embargo no se acata en la práctica y evidencia un doble discurso, el mismo que caracteriza a la modernidad.

c) Interculturalidad folklórica: se promueve en el contexto comercial, se hace uso y exaltación por los rasgos característicos de las culturas originarias agregando valor como bienes y servicios y constituye una exhibición de trajes, gastronomía, vestimentas o lenguas.

d) Interculturalidad con equidad: la implementada en reconocimiento de un Estado plurinacional; es decir, dar el justo valor a la autodeterminación de una unidad en la diversidad dentro del territorio estatal.

Es visible que este autor resalta que el Estado republicano, liberal y nacional ha impulsado históricamente la interculturalidad subordinada y folklórica, y de un tiempo para acá la institucionalizada, sobre todo en estos últimos gobiernos en los que inserta en el plano laboral a un indígena y se les puede apreciar en las recepciones de diferentes ministerios y entidades gubernamentales, lo cual evidencia un doble discurso en cuanto a sus derechos.

Sin embargo, en su posición de la interculturalidad con equidad se aprecia un planteamiento mucho más sólido y fuerte, en donde supone una relación compleja del reconocimiento de la unidad en diversidad dentro de una totalidad, aludiendo a un Estado, por lo que se aspira a un ejercicio pleno de la ciudadanía cultural o diversa, la cual recibe el reconocimiento y fortalecimiento de sus colectivos en respeto a su cosmovisión de la comunidad, naturaleza y ancestralidad.

Solamente desde esta apertura a la diferencia se legitiman los pueblos y nacionalidades indígenas para constituirse como parte esencial de un todo. Ese todo constituiría un Estado plurinacional. Esta orientación de vida y existencia marca una postura firme y es analizada también por Mignolo, citado en Walsh (2003: 9). Este sociólogo afirma que:

cuando la palabra interculturalidad la emplea el Estado, en el discurso oficial el sentido es equivalente a multiculturalidad [...]. El Estado quiere ser inclusivo, reformador, para mantener la ideología neoliberal y la primacía del mercado [...], en cambio el proyecto intercultural en el discurso de los movimientos indígenas está diciendo otra cosa, está proponiendo una transformación. No están pidiendo el reconocimiento y la inclusión en un Estado que reproduce la ideología neoliberal y el colonialismo interno, sino que están reclamando la necesidad de que el Estado reconozca la diferencia colonial (ética, política y epistémica).

Mignolo con esta declaración resalta lo que por muchos años no es comprendido, pues no se trata de que un Estado con derroche de sensibilidad reconozca a lo indígena, no. Es aceptar que los sistemas políticos, administrativos, sociales y económicos giren radicalmente hacia una sola dirección, a la aceptación de la diversidad. Esta postura implica el reconocimiento de un Estado plurinacional, pluritópico, vinculador de epistemes y sobre todo justo con todos y todas.

Dentro de este hilo conductor, y tomando las ideas reflexivas de Zúñiga (2011) acerca de la condición polisémica del término de interculturalidad, hace que sea mucho más cierto que esta noción se encuentra en la actualidad viviendo fuertes procesos de cambio, no nada más en nuestra América Latina, sino en el mundo.

El debate que se instala desde lo indígena cuestiona con fuerza la posición colonialista de los gobiernos latinoamericanos en la grave y deshumanizadora postura de desconocer al indígena como el otro, como el otro integrante del Estado, cuya diferencia hace resaltar la diversidad de nuestros pueblos.

\section{La interculturalidad como práctica social}

En la práctica de los pueblos indígenas radica probablemente una alternativa de solución a los gobiernos que buscan mejorar la calidad de vida de sus habitantes. Desde lo indígena un mundo mejor es posible, puesto que consiste en la recuperación del Estado de derecho y de la democracia de ciudadanos a partir de los derechos humanos. Es la administración de la muerte en función de la vida de los seres humanos y de la naturaleza, cuyo fundamento es una ética de la vida para el bien común, postulado en el que se ha fraguado las luchas de resistencia y de existencia en diversos contextos.

Es por ello que la interculturalidad como construcción imaginada y como práctica de convivencia se articula en la actualidad con un nuevo y amplio horizonte de sentido que en América Latina ha tomado forma en el proyecto político del buen vivir/vivir bien proclamado por los pueblos indígenas del Ecuador y de otros países suramericanos.

Es importante recalcar que esta propuesta no es retórica, es el modus vivendi del indígena. Es un modelo eminentemente comunitario con visión socialista, por poner un nombre, 
que se basa en el desarrollo de un nosotros comunitario en el cual todos los indígenas y no indígenas tengan cabida. Es un mundo del otro sustentado en la recuperación de la visión ancestral indígena en el espacio contemporáneo, como esperanza y nueva utopía posible (Hinkelammert y Mora, 2005: 395).

Esta utopía necesaria no busca un mundo perfecto, sino que es una fuente de vida y de esperanza para considerar la propuesta como un planteamiento con criterio fundamentado en la práctica y en el convencimiento del valor mismo de ese modelo de convivencialidad, reciprocidad, complementariedad y vincularidad (Prospecto UINPIAw, 2007).

Sin embargo, para comprender ese proceso histórico es importante recordar algunos episodios que marcaron la vida del hombre indígena en territorio ecuatoriano. Para ello se toma parte de las ideas exegéticas de Guerrero Arias (1993) en ese caminar del indígena hacia su renacimiento.

Este investigador señala que el enfrentamiento con el poder, representado por los terratenientes, no sólo imponía la necesidad de afirmación de una identidad social diferenciada para la negación, pues lo que estaba en juego era la tierra: centro vital para su reproducción tanto social como simbólica. Por esta razón constituye un hecho importante el que se plantee articularse a la sociedad dominante a partir de los propios elementos y recursos con los que contaban.

Con esto, aprender a leer o a escribir era prácticamente vital para no continuar siendo víctimas del blanco o criollo, y ya no ser engañados por los patrones o abogados, o cualquier otra persona constituyéndose un salto de vida en este proceso de afirmación de su conciencia étnica. Así pues, no es casual que a la lucha de la tierra, le sigue la lucha por la educación.

Solamente después de la caída de la hacienda, las comunidades indígenas comienzan a estructurarse como colectivos con una sistemática ganancia a la seguridad interna como grupo social que no se expresa en el desconocimiento del otro, sino en la forma de negociar con ese otro, ahora en condiciones distintas en su totalidad. El principio de que la tierra es suya y que fue conseguida con la lucha colectiva posibilita la estructuración ideológica necesaria para preservar y reproducir las veces que sean necesarias en pro de su nuevo presente.

Los indígenas desde la victoria contra la hacienda, que a la vez era contra el poder, mantienen la convicción de que mantenerse en resistencia o en insurgencia colectiva posibilitaron el triunfo y la transformación de su realidad de miseria, olvido, atraso y servidumbre en la que vivían.

En el Ecuador en la década de los setenta, en pleno despliegue de desarrollismo industrial, los estándares de analfabetismo en las comunidades indígenas eran insólitos.
La totalidad de esa población se encontraba sumergida en una parálisis paradigmática. Este fenómeno junto con la marginalidad social y económica frenó la capacidad creadora y el ejercicio de sus saberes y la pertinencia y buen uso de recursos tanto naturales como culturales.

Sin embargo, una de sus capacidades innatas sirve como el recurso emancipatorio en el uso del lenguaje. Dentro de la racionalidad del mundo indígena la comunicación no es una categoría abstracta, sino un recurso a través del cual se va generando una riqueza de significado que permite un nivel de vinculaciones sociales, donde el hombre más adulto es por lo general el más sabio y el que asume las orientaciones al colectivo.

Es así que con el diálogo comunitario se avanza en los análisis de su realidad social y en la búsqueda de las soluciones. Dialogar o "juntar cabezas" será siempre un acto creador, en el que cada cabeza socializa su saber y reactiva su carga de memoria para ponerla al servicio del presente y del porvenir.

En 1973 un pequeño colectivo de indígenas "juntan cabezas" e inician con la ayuda de la comunidad salesiana y la organización de voluntarios de Mato Grosso la creación de la primera escuela indígena en esos escenarios para atacar los altos indicadores de analfabetismo en las zonas, así como eliminar esas estadísticas por considerar que atentan contra la identidad cultural.

La primera escuela de educación básica que funcionó es a partir de 1973, cuyo docente fue un voluntario de Colegio Don Bosco, de Quito, Ecuador. Los siguientes años se extiende este proyecto y se crean más escuelas con docentes indígenas. Sin embargo, hasta la década de los ochenta empiezan a entender que no sólo es importante aprender a leer y escribir, es también por la educación en conjunto que ellos pueden superar otras limitantes dentro de sus comunidades.

\section{Construcción de una escuela con rostro propio}

La construcción de una escuela con rostro propio nacida desde la necesidad y conciencia del indígena debe evaluarse como el resultado de una nueva conciencia étnica, tal como lo afirma Guerrero Arias (1993).

Para estos indígenas la escuela y la orientación que asumen constituyen un recurso fundamental en su proceso histórico de insurgencias. Es desde donde se impulsa activamente el proceso de lucha por la reconquista de la tierra. Surge con un contenido ideológico distinto, pues cuestiona principios y estructuras en las que se ha sustentado el ejercicio del poder. La escuela toma otra orientación y trasciende su función instruccional. 
Según este autor, la escuela indígena surge para superar ese orden establecido por la sociedad moderna y es por eso que debía tener identidad propia. Entre sus principales rasgos se podrían deducir las siguientes características:

a) Es una respuesta comunitaria a las necesidades de la colectividad.

b) Es una estrategia para garantizar la vida.

c) Es en sí misma una forma de aprender en comunidad, ya que así es como son construidas.

d) Son la oportunidad perfecta de extensión y colaboración comunitaria, ya que es un proyecto autogestor.

e) Desde ella se crean otros núcleos productivos sociales: las bloqueras, cuyeras, tienda comunal, reforestación, panadería, caminos, acequias, capillas, entre otros.

f) Se convierte en el eje articulador y vinculador de la comunidad. Ahí se reúne la gente para "juntar cabezas".

$g$ ) Es el motivo para despertar las capacidades, talentos y fortalezas de sus miembros.

h) Representa el máximo valor de una comunidad. La organización atiende el bienestar colectivo.

Es de notar que el mayor triunfo que la escuela permite es afirmar la seguridad en sí mismos, aunado a la fuerza que da la organización comunitaria. Este resultado, al ver materializado los objetivos y la constatación de que en juntos podrían conseguir metas, fue propiciando con efectividad la confianza en la organización, en sus miembros y sobre todo en la importancia de tener una escuela en la comunidad.

Se evidencia que desde la concepción comunitaria, la escuela muestra ser un instrumento para la lucha social porque cumple una función política y conduce a una nueva acción insurgente si se quiere: la lucha por sus mejoras en la calidad de vida sin dependencias del poder económico del Estado, a pesar que éste tiene obligación en atenderlos; sin embargo, los miembros de la comunidad asumieron su propio bienestar a partir de la asamblea de hermanos, donde todos "juntan cabezas" y viven un propio sistema de gobierno basado en el respeto, unión y sentido del colectivo.

Además, en la escuela se refuerza lo que en la familia se vive, ya que los centros educativos son las mismas comunidades, en donde se aprende a sembrar, cosechar, escuchar al sabio, colaborar con los otros; en fin, la escuela es una extensión de la colectividad.

Como otro elemento preponderante en esta vida del indígena, en el rescate de su propio ser, está la recuperación de la palabra en su originalidad de lengua. En este sentido, estas escuelas no sólo representaron el eje de la develación indígena, sino también del resarcimiento de la lengua materna, en este caso el quichua.
Sin bien en la lucha por la tierra, la libertad, la educación afirmó una conciencia étnica muy importante, la lengua materna, enseñada desde la familia y reforzada en la escuela, es un elemento esencial para la memoria y retroalimentación de los saberes y eje de afirmación de su identidad cultural.

\section{Análisis prospectivo}

Esta panorámica social conduce a varias posturas. El elemento conductor de la interculturalidad bajo la mirada de la discriminación se podrá centrar por lo menos dos caminos:

a) El desarrollismo actual fundado en la civilidad del conocimiento sólo ha servido para el incremento material. El hombre del siglo xxI con toda la tecnología, la ciencia y la productividad a su alcance está cada vez más alejado de la condición humana y de su complejidad.

b) El aporte, avances y desarrollo de los pueblos indígenas en el territorio ecuatoriano como en nuestra América implica la formación de un hombre auténtico, de una convicción contundente en su cosmovisión.

Sin duda, a partir de estas reflexiones hay una notable aproximación para comprender la racionalidad del pensamiento indígena. Sus estrategias, dinámica y racionalidad producen cada vez más preguntas que respuestas, más llamados a la sensibilización social como al reconocimiento del otro como un auténtico otro.

Esta relación de poder marcada por la diferencia pautada desde la Colonia justifica esos paradigmas de discriminación y abuso histórico al hermano indígena. El discurso de la diferencia está fundado en los hilos de la promoción de una modernidad que promete desarrollo, comodidad y tecnología y evade otros saberes con profundidad epistemológica que podría convertirse en una postura de vida y de gobernabilidad.

Es de suma importancia recalcar que los pueblos originarios ecuatorianos viven francas encrucijadas frente a ese poder colonial. Aunque ellos sean constantes, perseverantes, organizados y con su convicción abya-yalista como su norte, viven una batalla todavía sin capítulo final.

\section{Reflexiones finales}

El sistema de escuela indígena asume un modelo o paradigma propio, desde su praxis y perspectiva intercultural, con una nueva dimensión ideológica y política comprometida y transformadora desde lo comunitario y que está vigente hasta nuestro días.

a) La gestación de una escuela dentro del contexto de la marginalidad, pobreza y olvido, denota un hombre y una mujer indígenas revolucionarios. Se atrevieron a rescatar su 
historicidad, su identidad, su lengua con el fin de ser reconocidos, como el otro, otro diferente, y digno igualmente a mí y a mis derechos humanos.

b) La perspectiva de una interculturalidad desde la equidad es el logro del indígena con el convencimiento de su valor desde la mismidad a la otredad. Es un ejemplo de ser en lo constructivo y valioso como actor y gestor social de su realidad. Concebir la educación intercultural desde el pensamiento de los pueblos originarios permite visualizarlo desde una mirada paradigmática abya-yalista que apertura el diálogo de saberes desde lo intercivilizatorio, dando respuesta comunitaria a las necesidades de un colectivo social.

c) Es un salto de vida para la formación del hombre y mujeres indígenas con consciencia de su valor de sabiduría, cultura y cosmología en general.

d) Es una estrategia de aprender desde la reciprocidad, ya que el pensamiento es compartido.

e) Es un proyecto autogestor desde el diálogo y vinculariedad de saberes.

f) El diálogo es el instrumento para despertar actitudes de un ethos que se traduce en capacidades y talento humano en convivencia.

$g$ ) Representa el máximo valor del vivir desde una filosofía propia del buen vivir.

h) Es la manifestación concreta que una orientación basada en la sabiduría transforma al hombre y a su comunidad.
Así pues, las posiciones determinantes en lo social, político y económico no son las alternativas de gobernabilidad. Siempre y cuando el líder esté fundado desde la humanidad, respeto y el bien común podrá vivirse una orientación en promoción del hombre, su talento y su dignidad.

Finalmente, es urgente de parte de los estados, gobernadores y ciudadanía en general optar por una postura digna, en la cual la diversidad, no discriminación, inclusión, equidad, dejen de ser elementos que representan solamente a una comunidad de intereses y pasar a la construcción real de comunidades que permitan y fortalezcan la convivencialidad y diversidad.

Los pueblos indígenas se orientan en dirección de la autonomía como un componente fundamental del bienestar. Al respecto, la autonomía se refiere al derecho a organizar su vida social, económica y política a partir de sus propias formas de organización y de herencia cultural, dentro de los estados nacionales en los cuales viven, por lo que a la academia le quedaría estudiar este sujeto como un modelo organizacional de trascendencia humana.

Sin duda, es de considerar que esa orientación social y consolidación de identidad está en proceso. El corazón de este fenómeno es esencialmente la educación básica con una acepción hacia la diversidad como un todo, a partir de la cual la inteligencia indígena se volvió al mundo. Por lo anterior, con base en estas evidencias que pretenden sensibilizar, afirma la autora, que desde lo indígena un mundo mejor es posible.

Referencias

Flores Galindo, A. (1988). Buscando un inca.

Identidad y utopia en los andes. La Habana:

Editorial Horizonte.

García, J. (2011). I Simposio Internacional de Interculturalidad desde el Abya Yala, Bárbula, Venezuela, 23 y 24 de febrero de 2012. Modalidad ponencia del vicerrector de la Universidad Indígena Amawtay Wasi Quito, Ecuador.

Guerrero Arias, P. (1993). El saber del mundo de

los cóndores. Identidad e insurgencia de la cultura andina. Quito: Ediciones Abya-Yala.

Hinkelammert, F. y Mora H. (2005). Hacia una economía para la vida. San José: DEI.

Moya, R. (2009). La interculturalidad para todos en América Latina, en L. E. Luis (ed.) (pp.21-56).

Sarango, L. (2010). La interculturalidad como práctica de vida y la educación superior como diálogo de saberes, en Las políticas públicas desde la visión de los pueblos indígenas. Videoforo realizado en el Paraninfo Daniel Ocluber Quirós el 26 de julio del 2010. San José de Costa Rica: Oficina de Audiovisuales unED.

Prospecto UinPIAW (2007) Prospecto Universidad de los Pueblos Indígenas Amawtay Wasi uinPIAW. Publicaciones semestrales.
Quito-Ecuador.

Walsh, C. (2003). Las geopolíticas del conocimiento y colonialidad del poder. Entrevista a Walter Mignolo. Polis, Revista de la Universidad Bolivariana de Santiago de Chile. Disponible en http:// redalcyc,uaemex.mex. Consultado el 20 de febrero de 2014.

Zúñiga, X. (2011). La interculturalidad como relación imaginada y práctica social: experiencias con y desde los pueblos indígenas en América Latina. Costa Rica: Publicaciones Cuadernos Intercambio. 